\title{
Soft Tissue Osteochondroma in Elbow Joint: A case report
}

\author{
Narula R, D Orth, MS, FAIMER, Arya A, MD, Verma LR, MS, Singh S, D Orth \\ Department of Orthopaedics, Rohilkhand, Medical College, Bareilly, India \\ Department of Pathology, Rohilkhand, Medical College, Bareilly, India
}

\begin{abstract}
A case of histopathologically verified soft tissue osteochondroma of the elbow joint is presented along with clinical and radiographic findings. The unique feature of this case is that the joint capsule was intact and the tumour was entirely extra-articular. A diagnosis of extraskeletal osteochondroma should be considered when a discrete ossified mass is localized in the soft tissue. Excision and biopsy were performed with complete resolution of the symptoms.
\end{abstract}

Key Words:

soft tissue tumour, chondroma, osteochondroma

\section{INTRODUCTION}

Surface lesions of bone usually present little diagnostic dilemma because the majority are conventional osteochondromas. Other surface bone lesions include periosteal chondroma, periosteal chondrosarcoma, and parosteal osteosarcoma. Mineralized soft tissue lesions such as myositis ossificans, synovial chondroma, and synovial sarcoma may present in a similar fashion when occurring in a juxtaarticular position. The soft tissue osteochondroma or para-articular osteochondroma may simulate some of these more aggressive tumours; thus, its recognition is important to avoid overtreatment. Here, we present a case of soft tissue osteochondroma of the elbow.

\section{CASE REPORT}

An 18-year-old male presented in the outpatient department with a history of swelling in his left elbow for the previous two years. There was no history of any trauma, fever, joint effusions or swelling in other joints. On examination, there was a spherical shaped swelling of the left elbow over the lateral aspect with no signs of inflammation. The swelling was more prominent in complete flexion, and measured approximately $2.5 \mathrm{~cm} X 2.5 \mathrm{~cm}$. On palpation, the swelling was bony hard and freely mobile. Range of motion of the elbow joint was restricted at the extremes by five to ten degrees.
Anteroposterior views of radiographic images showed patchy calcification, well demarcated on the lateral aspect of the elbow with partial overlapping onto the lateral condyle of the lower humerus and radial head. Lateral radiographic imaging revealed a globular calcified mass with no apparent connectivity to the bones of the elbow joint (Figure 1a and 1b). Routine laboratory investigations were conducted and results were within normal limits for erythrocyte sedimentation rate (ESR), serum uric acid, serum calcium, phosphorous, alkaline phosphatase, as well as renal and liver function tests.

The patient underwent an excisional biopsy (following application of a pneumatic tourniquet) under brachial block anaesthesia. A glistening white globular tumour was found in the soft tissues with no accompanying pedicle or connectivity to underlying bones. The joint capsule was intact and the tumour was extraarticular (Figure 2a). Gross examination showed a well-defined greyish brown globular mass measuring $5.5 \times 4.5 \mathrm{~cm}$ with firm to bony hard consistency. The cut surface showed solid variegated bony hard and soft areas (Figure 2b). Microscopic examination revealed endochondral ossification with a cartilage cap and mature trabecular bone underneath. Normal bone marrow elements were present in between bony trabeculae (Figures $3 \mathrm{a}$ and $3 \mathrm{~b}$ ). The diagnosis was soft tissue osteochondroma.

The postoperative phase was uneventful. Stitches were removed on the eighth postoperative day. A plaster slab cast was maintained at ninety degrees for three weeks, followed by gradual physiotherapy. On review at two months postoperatively, the patient had full range of motion in the joint.

\section{DISCUSSION}

A majority are conventional osteochondromas originating from the bone. Leung et al have reported a solitary osteochondroma in the elbow leading to acute dislocation of the joint in a young man. In that case, the tumour was present at the radio-capitellar articulation and dislocation was caused by trapping of the biceps tendon at the stalk of a solitary osteochondroma ${ }^{1}$. Similarly Karakurt et al have reported a solitary osteochondroma of the right elbow causing ulnar

Corresponding Author: Ramesh Narula, Associate Professor, Department of Orthopaedics, Rohilkhand Medical College, Bareilly (UP), India Email: rameshnarula55@gmail.com 


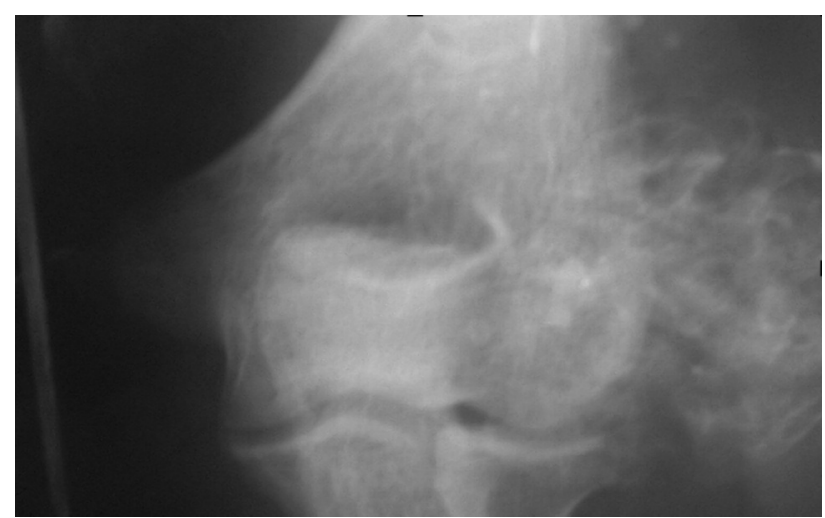

Fig. 1a: Anteroposterior radiographic view of affected elbow joint.

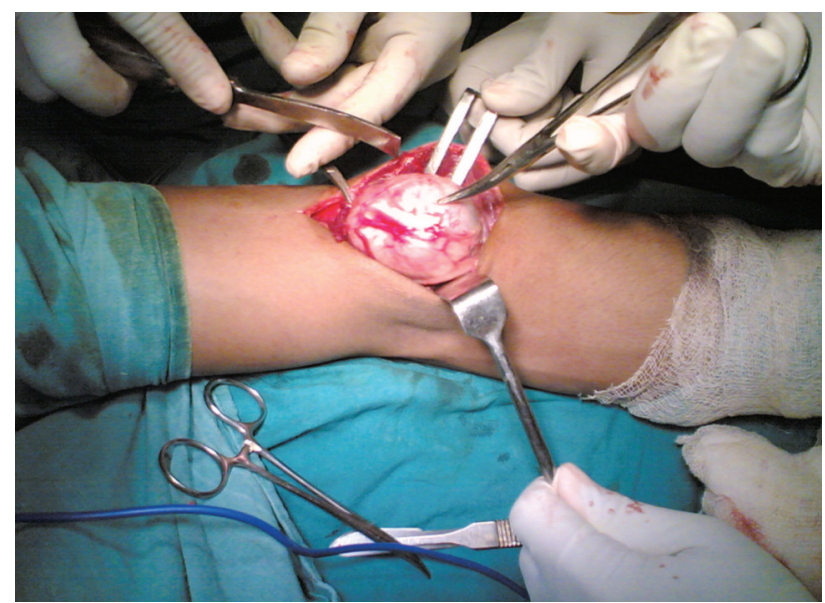

Fig. 2a: Intraoperative appearance of the tumour.

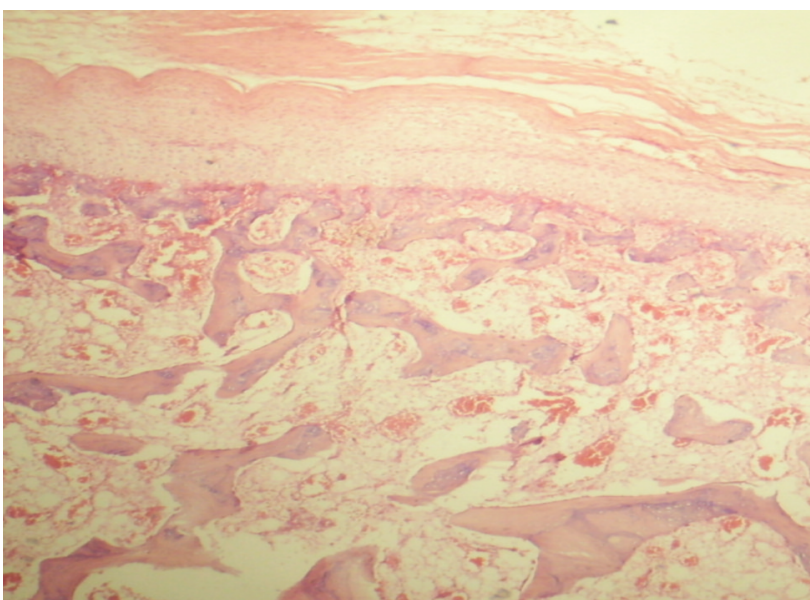

Fig. 3a: Low power magnification of tumour showing hyaline cartilage cap, endochondral calcification and bony trabeculae surrounded by fat and hematopoietic marrow.

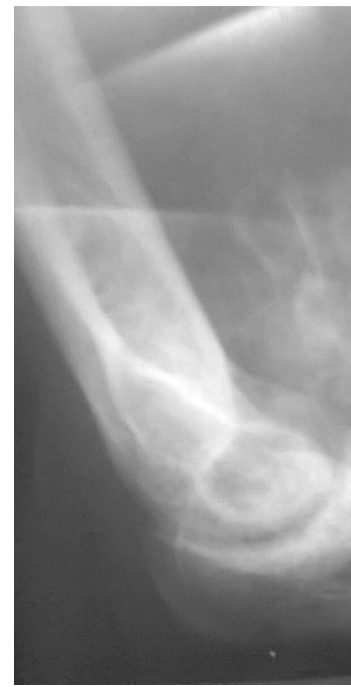

Fig. 1b: Radiograph lateral view.

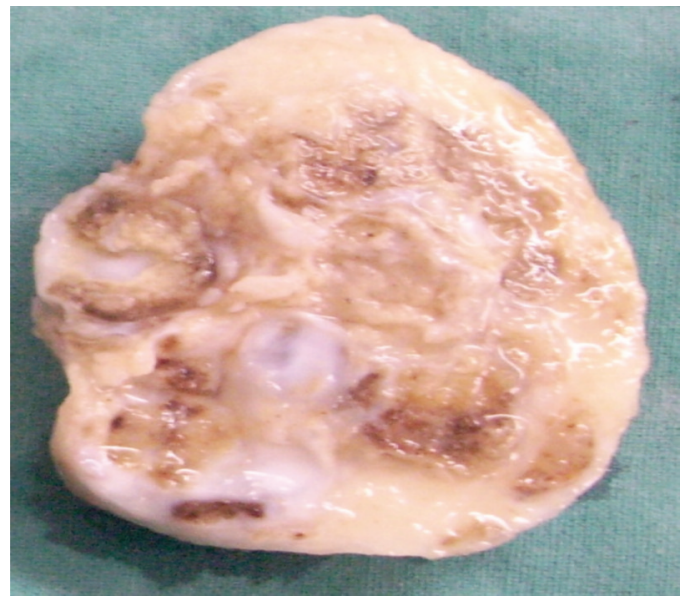

Fig. 2b: Gross specimen of soft tissue osteochondroma showing bony hard areas covered by thin cartilaginous caps and soft fatty areas.

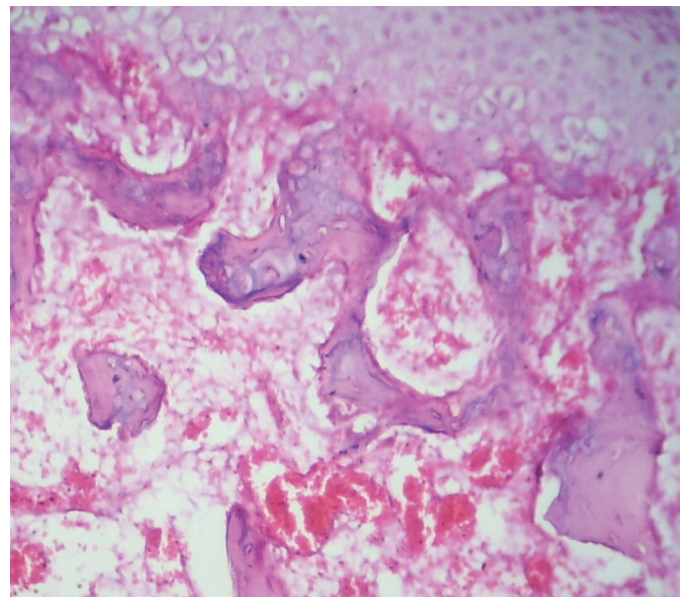

Fig. 3b: Photomicrograph showing hyaline cartilage cap, endochondral calcification and bony trabeculae with normal bone marrow. (hematoxylin and eosin ( $\mathrm{H} \& \mathrm{E}$ ) stained, $10 \mathrm{X}$ magnification). 
nerve compression. These osteochondromas originated from the olecranon and medial humeral epicondyle, respectively ${ }^{2}$.

Osteochondromas originating from the synovium have also been reported by other authors. For example, a synovial osteochondromatosis of the elbow was reported by Kamineni et al in twelve patients, all treated by synovectomy. Histological review showed that seven cases were primary and five were secondary osteochondromatosis ${ }^{3}$.

Overall, osteochondroma of soft tissue is a rarely occurring benign tumor. It has been reported in several tissues of the limbs and also in particular localizations (kidney, liver, tongue). Diagnosis is based on histological results, after elimination of the possibility of osseous chondroma or synovial osteochondroma ${ }^{4}$. Li and colleagues reported three cases of benign soft tissue osteochondromas with uncertain pathogenesis. Two cases were located in the subcutaneous tissues beneath the calcaneus. The third was located in the soft tissues near the left ankle joint. They suggest that diagnosis of soft tissue osteochondroma should be considered when a well-defined osseous mass is located in the soft tissues ${ }^{5}$. In the present case, the tumour was located at the elbow.

A definitive diagnosis of soft tissue osteochondroma is feasible using systemic approaches including histopathological examination, detailed physical examination and history, and radiological examination. Correct identification of this entity and differentiation from other mimicking conditions is crucial to avoid overtreatment. 


\section{REFERENCES}

1. Leung, YF, Ip SPS, Wong A, Ip WY. Acute dislocation of the elbow caused by a solitary osteochondroma of the proximal radius. Arch Orthop Trauma Surg. 2006; 126(2): 131-3

2. Karakurt L, Yilmaz E, Varol T, Ozdemir H, Serin E. Solitary osteochondroma of the elbow causing ulnar nerve compression: a case report. Acta Orthop Traumatol Turc. 2004; 38(4): 291-4.

3. Kamineni S, O'Driscoll SW, Morrey BF. Synovial osteochondromatosis of the elbow. J Bone Joint Surg [BR] 2002; 84(7): 961-6.

4. DeSmet L. Posterior interosseous neuropathy due to compression by a soft tissue chondroma of the elbow. Acta Neurol Belg. 2005; 105(2): 86-8.

5. Li C, Arger PH, Dalinka MK. Soft tissue osteochondroma. A report of three cases. Skeletal Radiol. 1989; 18(6): 435-7. 